\title{
$\begin{array}{ll}\text { Research Square } & \text { Preprints are preliminary reports that have not undergone peer review. } \\ \text { They should not be considered conclusive, used to inform clinical practice, }\end{array}$ or referenced by the media as validated information. \\ The Noninvasive Positive Airway Pressure Ventilation Therapy System: Modeling and MATLAB- based Investigating
}

YueYang Yuan ( $\nabla$ yuanyueyang@hncu.edu.cn )

Hunan City University

\section{Lixin XIE}

Chinese PLA General Hospital

\section{Li ZHOU}

Hunan Micomme Medical Technology Development Co., Ltd

\section{Xingshuo HU}

Chinese PLA General Hospital

Wei LIU

Hunan Micomme Medical Technology Development Co., Ltd

\section{Zheng DAl}

Hunan Micomme Medical Technology Development Co., Ltd

\section{Research Article}

Keywords: mechanical ventilation, noninvasive positive airway pressure, therapy system model

Posted Date: January 17th, 2022

DOI: https://doi.org/10.21203/rs.3.rs-1250352/v1

License: (1) This work is licensed under a Creative Commons Attribution 4.0 International License.

Read Full License 


\section{Abstract}

Background: The noninvasive positive airway pressure ventilation (NPPV) has been widely applied in therapy for the respiratory patient in acute respiratory distress syndrome (ARDS) and chronic obstructive pulmonary disease (COPD), etc.

Objective: In order to help clinician to investigate the technologies and measures of NPPV, a therapy system model included the respirator, breathing circuit and patient was designed.

Methods: Consequently, the mathematical sub-models of respirator, breathing circuit and mask, and respiratory airway were developed. With these sub-models, the ventilation system model of NPPV was prepared. The MATLAB-Based simulation experimental platform of NPPV system was set up to conduct the simulation experiments. In addition, by using the active servo lung ASL5000, a physical experiment platform was designed for comparative tests. Both of simulated experiments and physical experiments were operated with the simulated patient in ARDS, COPD and patient without spontaneous breathing.

Results: The simulated experimental outputs are coincident with the physical experimental outputs ( $\mathrm{P} \geq$ 0.31 with TTEST, $R \geq 0.92$ with CORREL).

Conclusions: The experimental results substantiated that the therapy system model of NPPV is operational and workable. With this system model, it will benefit clinicians and researchers to conveniently promote the development of NPPV.

\section{Background}

The respiratory diseases seriously threaten human health and life now. In the world, there are more than 3 millions of patients (more than $6 \%$ of the total deaths) a year die of the chronic obstructive pulmonary disease (COPD) alone ${ }^{[1]}$. The common respiratory diseases mainly include COPD, acute respiratory distress syndrome (ARDS), respiratory failure, etc. ${ }^{[2-4]}$. The therapies for patients in respiratory diseases are often assisted with the mechanical ventilation (MV). Since the infectious respiratory diseases appear, such as severe acute respiratory syndrome (SARS) and corona virus disease 2019 (COVID-19), the MV has been an important role in fighting against the pandemic and curing the infected patient ${ }^{[5]}$. Now among the popular measures in MV, the noninvasive positive airway pressure ventilation (NPPV) is one of the most widely applied and effective ventilation ${ }^{[6-7]}$.

In order to reduce or avoid the risks of ventilation in clinical trials, throughout the years, many trials and observational studies were analyzed to determine what ventilator parameters would benefit or injury the respiratory patient ${ }^{[8-9]}$. The virtual experimental ventilation is an operational measure for preclinical evaluation before the application of MV in patient. The development of ventilation system model of NPPV based on MATLAB is one of the most convenient and feasible measures. Usually, the respiratory airway model in resistance and compliance has been used to investigate the gas exchanging between the lung and atmosphere based on the electric circuit ${ }^{[10-11]}$. But so far, there are few introduce about the 
simulation therapy system model of NPPV which includes the sub-model of ventilation device (respirator) and the sub-model of respiratory patient.

Consequently, in the programming condition of MATLAB Simulink, a simulated experimental platform of NPPV was developed for conducting a serial of simulated ventilation experiments with the simulated respiratory patients in COPD, ARDS and patient without spontaneous breathing (SB). This developed system model of NPPV will conveniently promote developing the technologies in NPPV.

\section{Results}

After running simulation ventilation with a developed NPPV system therapy system more than 10 minutes, the results displayed in the simulation oscilloscope were collected as illustrated in Fig•1 (a), (b) and (c) which respectively demonstrate the experimental data curves for ventilating the patients in COPD, ARDS and patient without SB.

The simulated respiratory airflow (Qa_sim), pressure in lung (PI_sim) and tidal volume (Vt_sim) were respectively compared with the actual respiratory airflow (Qa_act), pressure in lung (PI_act) and tidal volume (Vt_act). As illustrated in Fig •2, here both of the simulation experimental output curves and the physical experimental output curves are coincident.

By using the TTEST algorithm and CORREL algorithm in EXCEL to analyze both groups of data collected in simulated experiments and physical experiments, the results of $P$ and $R$ were listed in Tab•1. $P \geq 0.31$ means the differences between both groups of data are not obvious. $R \geq 0.92$ means both are in good consistency.

\section{Tab•1}

the P-value and R-value between both groups of output data in simulated and physical experiments

\begin{tabular}{|lllllll|}
\hline \multirow{2}{*}{ Data Name } & \multicolumn{3}{c}{ P-value with TTEST } & \multicolumn{3}{c|}{ R-value with CORREL } \\
\cline { 2 - 7 } & COPD & ARDS & Without SB & COPD & ARDS & Without SB \\
\hline Respiratory flow & 0.77 & 0.77 & 0.65 & 0.97 & 0.92 & 0.97 \\
\hline Pressure in lung & 0.62 & 0.31 & 0.55 & 0.99 & 0.98 & 0.99 \\
\hline Tidal volume & 0.78 & 0.92 & 0.75 & 0.99 & 0.97 & 0.99 \\
\hline
\end{tabular}

\section{Discussion}

The simulation system model of NPPV is convenient and safe for researchers and clinicians to develop the technologies in NPPV. For example, more ventilation modes will be possibly proposed with setting hoped-for conditions and parameters in simulation experiments. With the simulation system model of NPPV, both of the respirator and respiratory patient can be simulated. Not only the ventilation conditions 
but also the pathological features of the respiratory patient will conveniently be investigated. Additionally, if necessary in the next investigations, the sub-model of respiratory airway can be optimized with other

model such as Resistance-Inertance-Compliance (RIC) model, augmented RIC model, etc ${ }^{[17]}$.

One of the main purposes of NPPV is to promote the respiratory patient to inhale sufficient flash air into the lung and exhale carbon dioxide $\left(\mathrm{CO}_{2}\right)$ out the lung thoroughly. The respiratory flow and tidal volume (Vt) are usually considerable indexes to decide the effective ventilation. The adequate $\mathrm{Vt}$ usually ranged in $8 \sim 10 \mathrm{~mL} \cdot \mathrm{kg}^{-1}$ (or $400 \sim 800 \mathrm{~mL}$ ) is one of the major prerequisites for normal breathing. Adequate leak volume also is an important prerequisite to avoid $\mathrm{CO}_{2}$ retention happened in NPPV ${ }^{[18]}$. When the leak volume (Vleak) is less than the $\mathrm{Vt}$, it is easy to cause that the $\mathrm{CO}_{2}$ and other waste gases cannot be completely discharged, and can be inhaled again by patient. Therefore, the leak flow and leak volume were listed in the simulated experimental results for conveniently observing and determining the ventilation effect.

In NPPV, a high pressure and a large volume often possibly cause a pulmonary barotraumas such as tension pneumothorax, mediastinal emphysema, pulmonary emphysema, and so on. But a low pressure and a small volume often possibly cause the respiratory patient in defective ventilation ${ }^{[19]}$. Based on the simulation ventilation system model of NPPV mentioned in this paper, the respiratory mechanical parameters such as the volume of $\mathrm{Vt}$ and pressure of Plung can be observed. Well investigations and observations though the simulated experiments operated in the system model will be beneficial to determine the setting of relevant NPPV parameters, and to reduce the possibility of injuries to patient.

Respiratory disease is one of the greatest threaten to human health and life safety. In present, one of most common therapy measures is to use NPPV with respiratory patient. Especially for the patient in severe acute respiratory syndromes (SARS), H7N9 avian influenza or Corona Virus Disease in 2019 (COVID-19), clinicians are more aware of the importance of treatment with NPPV ${ }^{[20]}$. The system model of NPPV developed in this paper includes the sub-model of respirator, the sub-model of breath circuit and nasal mask, and the sub-model of respiratory model. It is helpful for setting ventilation conditions and parameters to simulate the NPPV according to the pathological characteristics and symptoms appeared in different respiratory patients.

\section{Conclusion}

A simulation ventilation system model of NPPV including respirator, breathing circuit, oral-nasal mask and respiratory patient was developed based on MATLAB. The NPPV was carried out with the simulated patients in COPD and ARDS and patient without spontaneous breathing. By comparing the results from the simulated experiments and from the ASL5000-based physical experiments, the output data in both groups of experiments are consistent with the no obvious difference $(P \geq 0.31)$ and high consistency ( $R$ $\geq 0.92$ ). And the experimental results illustrated that the developed simulation system model of NPPV is feasible to simulation ventilation with the respiratory patients. This system model of NPPV will be helpful 
to reduce or avoid the risks of clinical trials, and to provide the premeditated measure for NPPV applied with the respiratory patient.

\section{Methods}

\section{Modeling for Ventilation Therapy System}

As shown in Fig $\bullet$, the ventilation therapy system of NPPV is composed of a NPPV respirator, a breathing circuit and a mask (oral-nasal mask/ or nasal mask, etc), and a respiratory patient. By using MATLAB version 9 (R2016a), the ventilation system model was designed with developing a sub-model of respirator, a sub-model of breaching circuit and mask, and a sub-model of respiratory airway.

The purpose of a NPPV respirator is to deliverer a positive pressure into the patient's airway to support his breathing. This pressure ensures that the patient's respiratory airway is unblocked. The airflow freely moves in the airway under the positive pressure. And then the gas is exchanged between the lung and the atmosphere. The common ventilation methods in NPPV respirator usually include pressure control ventilation (PCV) and (PSV). PCV/PSV is usually applied in the patients without/with spontaneous breathing.

As shown in Equ $\bullet$, it describes the output pressure of $P_{\mathrm{a}}$ from the respirator when the respirator is working in the PCV mode. In the period of inspiration $\left(T_{1}<\mathrm{t}<T_{\mathrm{E}}\right)$, the pressure of $P_{\mathrm{a}}$ exponentially increases from the expired positive airway pressure (EPAP) to inspired positive airway pressure (IPAP). In the period of expiration $\left(T_{\mathrm{E}}<t \leq T\right)$, the pressure of $P$ a exponentially decreases from the IPAP to EPAP. Here $\tau_{1}$ and $\tau_{2}$ respectively expresses the time constant of rising and falling.

$$
\left\{\begin{array}{l}
P_{\mathrm{a}}=(\mathrm{IPAP}-\mathrm{EPAP}) \times\left(1-\mathrm{e}^{-\frac{t}{\tau_{1}}}\right)+\operatorname{EPAP} \quad\left(T_{\mathrm{I}}<t<T_{\mathrm{E}}\right) \\
P_{\mathrm{a}}=(\mathrm{IPAP}-\mathrm{EPAP}) \times\left(\mathrm{e}^{-\frac{t-T_{\mathrm{E}}}{\tau_{2}}}\right)+\operatorname{EPAP} \quad\left(T_{\mathrm{E}}<t<T\right)
\end{array}\right.
$$

As shown in Equ•2, it describes the output pressure of $P_{\mathrm{a}}$ when the respirator is working in PCV mode. The respirator is triggered to output IPAP or EPAP at the time of $T_{\text {Itrig }}$ or $T_{\text {Etrig }}$ which deputy the start of inspiration or expiration. The ventilation cycle of $T$ is the time span from current $T_{\text {Itrig }}$ to next $T_{\text {ltrig, }}$ or from current $T_{\text {Etrig }}$ to next $T_{\text {Etrig. }}$. In the period of inspiration or expiration, $P_{\mathrm{a}}$ exponentially increases from EPAP to IPAP or decreases from IPAP to EPAP. 


$$
\begin{cases}P_{a}=(\mathrm{IPAP}-\mathrm{EPAP}) \times\left(1-\mathrm{e}^{-\frac{t}{\tau_{1}}}\right)+\mathrm{EPAP} \quad\left(T_{\text {Itrig }}<t \leq T_{\text {Etrig }}\right) \\ P_{a}=(\mathrm{IPAP}-\mathrm{EPAP}) \times\left(\mathrm{e}^{-\frac{t-T_{\text {Etrig }}}{\tau_{2}}}\right)+\mathrm{EPAP} \quad\left(T_{\text {Etrig }}<t \leq T\right)\end{cases}
$$

With the NPPV, the airflow from the respirator is delivered to the patient's airway though the breathing circuit and mask. The airflow in the circuit and mask is described in the Equ 3 . Where $Q_{m}$ deputies the airflow provided by the respirator. $Q_{\text {leak }}$ deputes the leak flow which flows to the atmosphere through the leak hole in the mask. $Q_{\text {in }}$ and $Q_{\mathrm{ex}}$ respectively depute the inspiratory airflow and the expiratory airflow. During inspiration, the $Q_{\mathrm{m}}$ is separated into $Q_{\text {leak }}$ and $Q_{\mathrm{in}}$. During the patient's expiration, both of $Q_{\mathrm{m}}$ and $Q_{\text {ex }}$ flow to the atmosphere as $Q_{\text {leak }}$. The leak flow of $Q_{\text {leak }}$ is decided by the pressure of $P_{\mathrm{a}}$ in the mask as described in Equ•3. Where $a, b$ are the coefficients of the exponential function.

$$
\left\{\begin{array}{llll}
Q_{\mathrm{m}}=Q_{\text {leak }}+Q_{\text {in }} & \left(T_{\mathrm{I}}<t \leq T_{\mathrm{E}}\right. & \text { or } & \left.T_{\text {Itrig }}<t \leq T_{\text {Etrig }}\right) \\
Q_{\text {leak }}=Q_{\mathrm{m}}+Q_{\text {ex }} & \left(T_{\mathrm{E}}<t \leq T\right. & \text { or } & \left.T_{\text {Etrig }}<t \leq T\right) \\
Q_{\text {leak }}=-\mathrm{a} \times\left(P_{\mathrm{a}}\right)^{\mathrm{b}} & & &
\end{array}\right.
$$

As shown in Fig•4(a), the human respiratory airway includes the respiratory track and the lung. The viscous resistance in the respiratory track and the pulmonary elastic force (or compliance) in the lung prevent the airflow moving. For easily understood, the viscous resistance in track $\left(R_{\mathrm{a}}\right)$ and the compliance in lung $\left(C_{\mathrm{L}}\right.$, reciprocal of the elastic force) are usually analogized as the equivalent electric resistance and the equivalent electric capacitance.

In Equ 4 , it describes the pressure of $P_{\mathrm{a}}$ mathematically related to $Q_{\mathrm{a}}, R_{\mathrm{a}}$ and $C_{\mathrm{L}}$. Here $R_{\mathrm{a}}$ represents the viscous resistances in the respiratory track. $C_{\mathrm{L}}$ represents the compliance in the lung. $Q_{\mathrm{a}}$ represents the airflow in the airway. In the period of inspiration $\left(T_{\mathrm{l}}<\mathrm{t} \leq T_{\mathrm{E}}\right), Q_{\mathrm{a}}=Q_{\mathrm{in}}$, and $R_{\mathrm{a}}=R_{\mathrm{in}}, Q_{\mathrm{in}}$ enters into the lung. In the period of expiration $\left(T_{\mathrm{E}}<\mathrm{t} \leq T\right), Q_{\mathrm{a}}=Q_{\mathrm{ex}}$, and $R_{\mathrm{a}}=R_{\mathrm{ex}}, Q_{\mathrm{ex}}$ exits from the lung. 


$$
\left\{\begin{array}{l}
P_{\mathrm{a}}=R_{\mathrm{a}} \times Q_{\mathrm{a}}+\frac{1}{C_{\mathrm{L}}} \int_{0}^{T}\left(Q_{\mathrm{a}}\right) d t \\
R_{\mathrm{a}}= \begin{cases}R_{\text {in }} & \left(T_{\mathrm{I}}<t<T_{\mathrm{E}}\right) \\
R_{\text {ex }} & \left(T_{\mathrm{E}}<t<T\right)\end{cases} \\
Q_{\mathrm{a}}= \begin{cases}Q_{\text {in }} & \left(T_{\mathrm{I}}<t<T_{\mathrm{EI}}\right) \\
Q_{\text {ex }} & \left(T_{\mathrm{E}}<t<T\right)\end{cases}
\end{array}\right.
$$

In the period of inspiration, the contracted respiratory muscles and the expanded chest cavity result a negative pressure in the lung, and the spontaneous inspiratory flow is inhaled into the lung. In the period of expiration, the relaxed respiratory muscles and the recovered chest cavity result a positive pressure in the lung, and the spontaneous expiratory flow is exhaled out of the lung. In Equ•5, it expresses the inspiratory effort pressure of $P_{\mathrm{m}}$ produced by respiratory muscle working ${ }^{[12-13]}$. In Fig.4(b), the $P_{\mathrm{m}}$ is a mathematic function of time $(t)$ which ranges in a respiratory cycle $(0<t \leq T) . P_{\mathrm{m}}$ is in decreasing $(0<\mathrm{t}$ $\left.\leq T_{\text {pm_rise }}\right)$, holding $\left(T_{\text {pm_rise }}<t \leq T_{\text {pm_hold }}\right)$, releasing $\left(T_{\text {pm_hold }}<t \leq T_{\text {pm_release }}\right)$, and zero $\left(T_{\text {pm_release }}<t\right.$ $\leq T)$.

$$
P_{\mathrm{m}}= \begin{cases}-P_{\mathrm{m}_{-} \text {max }} \times \sin \left(\frac{2 \pi t}{4 \times T_{\mathrm{pm} \_ \text {rise }}}\right) & \left(0<t \leq T_{\mathrm{pm} \_ \text {rise }}\right) \\ -P_{\mathrm{m} \_ \text {max }} & \left(T_{\mathrm{pm} \_ \text {rise }}<t \leq T_{\text {pm_hold }}\right) \\ -P_{\mathrm{m} \_ \text {max }} \times \sin \left(\frac{\pi}{2}+\frac{2 \pi\left(t-T_{\mathrm{pm} \_ \text {hold }}\right)}{4 \times\left(T_{\mathrm{pm} \_ \text {release }}-T_{\mathrm{p} \_ \text {hold }}\right)}\right) & \left(T_{\mathrm{pm} \_ \text {hold }}<t \leq T_{\mathrm{pm} \_ \text {release }}\right) \\ 0 & \left(T_{\mathrm{pm} \_ \text {release }}<t \leq T\right)\end{cases}
$$

\section{Simulated Ventilation}

As illustrated in Fig •5, with the programming environment in MATLAB simulink, a simulation experimental platform was designed. In this platform, the function module (FCN) of "Respirator" is designed to perform the function of Equ•1 and Equ•2. The time constants of $\tau_{1}$ and $\tau_{2}$ in Equ•1 and Equ 2 were taken as $0.1 \mathrm{~s}$ and 0.05 s respectively. The FCN of "Leakage" was designed to perform the function of Equ•3. The sub-model of respiratory track and lung was designed with an adjustable electric 
resistance $\left(R_{\mathrm{a}}\right)$ and an adjustable capacitance $\left(C_{\mathrm{L}}\right)$ to perform the function of Equ•4. The FCN of "Spontaneous Breathing" was designed to perform the function of Equ•5.

The parameters in the respirator mainly includes IPAP, EPAP, breath rate (BPM), the ratio of inspiration time vs expiration time (IE), working mode (Mode) and the airflow of triggered threshold (Threshold). The respiratory parameters in the model of respiratory airway mainly includes inspiratory resistance (Rin), expiratory resistance (Rex), lung compliance (CL), max inspiratory effort pressure (Pm_max), the ratio of inspiratory effort pressure in decreasing time (Pm_riese), holding time (Pm_hold) and releasing time (Pm_release). Secondly, the simulated experiments were carried to collect and observe the experimental results. In the virtual oscilloscope, the data curves were displayed.

PCV and PSV were designed in the model of NPPV respirator. "Mode $=0$ " means the PCV was set to support the breathing of the patient without spontaneous breathing. "Mode $=1$ " means the PSV was set for the patient in ARDS or COPD. In PSV, when the respiratory airflow proved by spontaneous breathing reaches the threshold, the respirator will be triggered to output IPAP or EPAP. The simulated breathing circuit meets the standard breathing circuit in size of $\varphi 2.2 \mathrm{~cm} \times L 180 \mathrm{~cm}$. The simulated mask was designed to meet the oral-nasal mask (Bestfit-2M, curative Medical Inc, Santa Clara, CA). In Equ•4, the coefficients were set with $a \approx 0.2$ and $b \approx 0.65$. Setting the parameters of system model as shown in $\mathrm{Tab} \cdot 2^{[14-16]}$, the simulated respiratory patient in COPD or ARDS or without SB was proposed to be ventilated with the simulated respirator.

\section{Tab•2}

\section{the set parameters in respirator and patients}




\begin{tabular}{|c|c|c|c|c|}
\hline \multirow[t]{2}{*}{ Parameters } & & \multicolumn{3}{|c|}{ Respiratory patient } \\
\hline & & COPD & ARDS & Without SB \\
\hline \multirow[t]{6}{*}{ In respirator } & IPAP $₫ \mathrm{cmH}_{2} \mathrm{O}$ & 20 & 12 & 15 \\
\hline & $\mathrm{EPAP} \llbracket \mathrm{cmH}_{2} \mathrm{O} \rrbracket$ & 5 & 4 & 4 \\
\hline & $\mathrm{BPM} \otimes \mathrm{b} \cdot \mathrm{min}^{-1} \square$ & - & - & 15 \\
\hline & IE & - & - & 0.67 \\
\hline & Model & PSV & PSV & PCV \\
\hline & Threshold $\otimes L \cdot \min ^{-1} \square$ & 2 & 2 & 0 \\
\hline \multirow[t]{8}{*}{ In patient } & $\mathrm{Rin} \nabla \mathrm{cmH}_{2} \mathrm{O} \cdot \mathrm{s} \cdot \mathrm{L}^{-1} \mathrm{\square}$ & 21 & 11 & 6 \\
\hline & $\mathrm{Rex} \bigotimes \mathrm{cmH}_{2} \mathrm{O} \cdot \mathrm{s} \cdot \mathrm{L}^{-1} \square$ & 23 & 16 & 6 \\
\hline & $\mathrm{C}\left[\mathrm{mL} \cdot\left(\mathrm{cmH}_{2} \mathrm{O}\right)^{-1}\right]$ & 53 & 30 & 50 \\
\hline & $\mathrm{Pm} \_\mathrm{max} \rrbracket \mathrm{cmH}_{2} \mathrm{O} \cdot \mathrm{s} \cdot \mathrm{L}^{-1} \mathrm{\square}$ & 24 & 21 & 0 \\
\hline & $\mathrm{bpm} \otimes \mathrm{b} \cdot \mathrm{min}^{-1} \square$ & 18 & 25 & 0 \\
\hline & Pm_rise $\% \bigotimes$ & 35 & 27 & 0 \\
\hline & Pm_hold $\nabla \% \bigotimes$ & 0 & 0 & 0 \\
\hline & Pm_release $₫ \% \rrbracket$ & 23 & 20 & 0 \\
\hline \multicolumn{5}{|c|}{ Note: $T_{p m \_r i s e}=P m \_r i s e \% \times T, T_{p m \_h o l d}=\left(P m \_r i s e+P m \_h o l d\right) \% \times T$. } \\
\hline
\end{tabular}

In order to prove the practicability of the simulation ventilation system of NPPV, one of best measures is to check the simulation results with physical experimental results. As shown in Fig•6, a physical experimental platform was designed with mainly using the active servo lung ASLI5000 (IngMar medical, USA) and the noninvasive respirator ST-30k (Hunan Micomme medical, China). The respirator of ST-30K was connected to the head model though a breathing circuit $(d 2.2 \mathrm{~cm} \times / 180 \mathrm{~cm})$ and a oral-nasal mask (Bestfit-2M). A simulated upper airway was inserted into the head model. And the upper airway was connected to the $S L 5000$ though a breathing circuit $(d 2.2 \mathrm{~cm} \times / 70 \mathrm{~cm})$. On the base of physical experimental platform, also setting the same parameters as listed in Tab•1, a serial of physical experiments were conducted.

\section{Abbreviations}


NPPV: noninvasive positive airway pressure ventilation;

COPD: chronic obstructive pulmonary disease;

COVID-19: corona virus disease 2019;

MV: Mechanical ventilation;

Qleak: leak flow;

Plung: intrapulmonary pressure;

Vt: tidal volume;

Qa_sim: simulated respiratory airflow;

Vt_sim: simulated tidal volume;

Vt_act: actual tidal volume;

RIC: resistance-inertance-compliance;

PSV: pressure support ventilation;

IPAP: inspired positive airway pressure;

IE: the ratio of inspiration time vs expiration time;

Rex: expiratory resistance;

Pm_max: max inspiratory effort pressure;

Tpm_rise: the time of inspiratory effort pressure in decreasing;

Tpm_hold: the time of inspiratory effort pressure in holding;

Tpm_release: the time of inspiratory effort pressure in releaseing;

Pm_riese; the ratio of inspiratory effort pressure in decreasing time;

Pm_hold: the ratio of inspiratory effort pressure in holding time;

Pm_release: the ratio of inspiratory effort pressure in releasing time;

\section{Declarations}

\section{Availability of data and materials}

Not applicable.

\section{Competing interests}

No potential conflict of interest was reported by the authors. 


\section{Funding}

Hunan Provincial Natural Sciences Funding Project, China\2020JJ41598区Key research program of Hunan

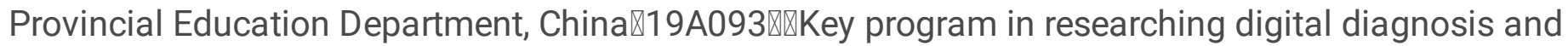
treatment equipment supported by the Ministry of Science and Technology of China『2021YFC0122500》

\section{Authors' contributions}

YUAN YueYang wrote this paper. ZHOU Li review the English. XIE Lixin and HU Xingshuo checked the respiratory data.

LIU Wei prepared physical experiments. DAI Zheng prepared the NPPV respirator.

\section{References}

1. Soriano J B, Kendrick P J, Paulson K R, et al. Prevalence and attributable health burden of chronic respiratory diseases, 1990-2017: a systematic analysis for the Global Burden of Disease Study 2017 - Science Direct [J]. The Lancet Respiratory Medicine, 2020, 8(6):585-596.

2. Carpagnano $G$ E, Sabato R, Lacedonia $D$, et al. New non invasive ventilator strategy applied to COPD patients in acute ventilator failure [J]. Pulmonary pharmacology \& therapeutics, 2017, 46(4): 64-68.

3. Momtaz O, El-Khateeb A, Amin E. Study of non-invasive mechanical ventilation in ICU patients: clinical and prognostic relevance [J]. Fayoum University Medical Journal, 2019, 2(1): 37-46.

4. David-Joao P G, Guedes M H, Rea-Neto A, et al. Noninvasive ventilation in acute hypoxemic respiratory failure: A systematic review and meta-analysis [J]. Journal of critical care, 2019, 49(1): 84-91.

5. Salah H M, Mehta J L. Meta-analysis of the effect of colchicine on mortality and mechanical ventilation in COVID-19 [J]. The American Journal of Cardiology, 2021, 145(2):170-172.

6. Kopp W, Gedeit R G, Asaro L A, et al. The Impact of Preintubation Noninvasive Ventilation on Outcomes in Pediatric Acute Respiratory Distress Syndrome [J]. Critical Care Medicine, 2021, 49(5): 816-827.

7. Killeen B M, Wolfson A B. Noninvasive positive pressure ventilation for cardiogenic pulmonary edema [J]. Academic Emergency Medicine, 2020, 27(12): 1358-1359.

8. Al-Naggar N, Al-Hetari H, Al-Akwaa F. Simulation of Mathematical Model for Lung and Mechanical Ventilation[J]. Journal of Science and Technology, 2016, 21(1):1-11.

9. Yuriditsky E, Horowitz J M, Nair S, et al. Simulation-based up training improves provider comfort in the management of critically ill patients with COVID-19[J]. Journal of Critical Care, 2021, 61:14-17.

10. LIU Tianya, QIAO Huiting, LI Deyu, at el. Simulation of spontaneous breathing for healthy adults using a nonlinear airway-segmented model of respiratory mechanics [J]. Journal of Biomedical Engineering, 2019, 36(1):107-112,121. (Chinese) 
11. Sammour I A, Chatburn R L. Inspiratory pressure rise time, ventilator hardware, and software influence regional ventilation in a simulated bronchopulmonary dysplasia lung model [J]. Respiratory Care, 2021, 66(5): 751-757.

12. Esra Saatçi, Aydin Akan. Lung model parameter estimation by unscented kalman filter [C]. Annual International Conference of the IEEE Engineering in Medicine and Biology Society. Cite Internationale, Lyon, France. August 23-24, 2007:2556-2559

13. Yoshitsugu Yamada, Hong-Lin Du. Analysis of the mechanisms of expiratory asynchrony in pressure support ventilation: a mathematical approach [J]. Journal of Applied Physiology, 2000, 88(6):21432150.

14. Chen Yuqing, Yuan Yueyang, Zhang Hai, at el. Estimated calculation of expiratory time constants for expiratory resistance evaluation during noninvasive positive pressure ventilation [J]. Chinese Journal of Biomedical Engineering, 2020, 26(05):385-391. (Chinese)

15. Arnal J M, Garnero A, Saoli M, et al. Parameters for simulation of adult subjects during mechanical ventilation [J]. Respiratory Care, 2018, 63(2): 158-168.

16. Dexter A, Mcninch N, Kaznoch D, et al. Validating Lung Models Using the ASL 5000 Breathing Simulator [J]. Simulation in Healthcare Journal of the Society for Simulation in Healthcare, 2018, 13(2):117-123.

17. Diong B, Rajagiri A, Goldman M, et al. The augmented RIC model of the human respiratory system [J]. Medical \& Biological Engineering \& Computing, 2009, 47(4):395-404.

18. Poon C S, Tin C, Song G. Submissive hypercapnia: Why COPD patients are more prone to $\mathrm{CO}_{2}$ retention than heart failure patients [J]. Respiratory Physiology \& Neurobiology, 2015, 216(9):86-93.

19. Yang YiYi, Yao ShangLong, Shang You. New progress of pathogenesis in ventilator-induced lung injury [J]. Chinese Critical Care Medicine, 2016, 28(9):861-864. (Chinese)

20. Song Yuanlin, Bai Chunxue. Shanghai expert consensus for respiratory clinic quality control during epidemic 2019-nCoV time [J]. Fudan University Journal of Medical Sciences, 2020, 47(2): 143-150. (Chinese)

\section{Figures}



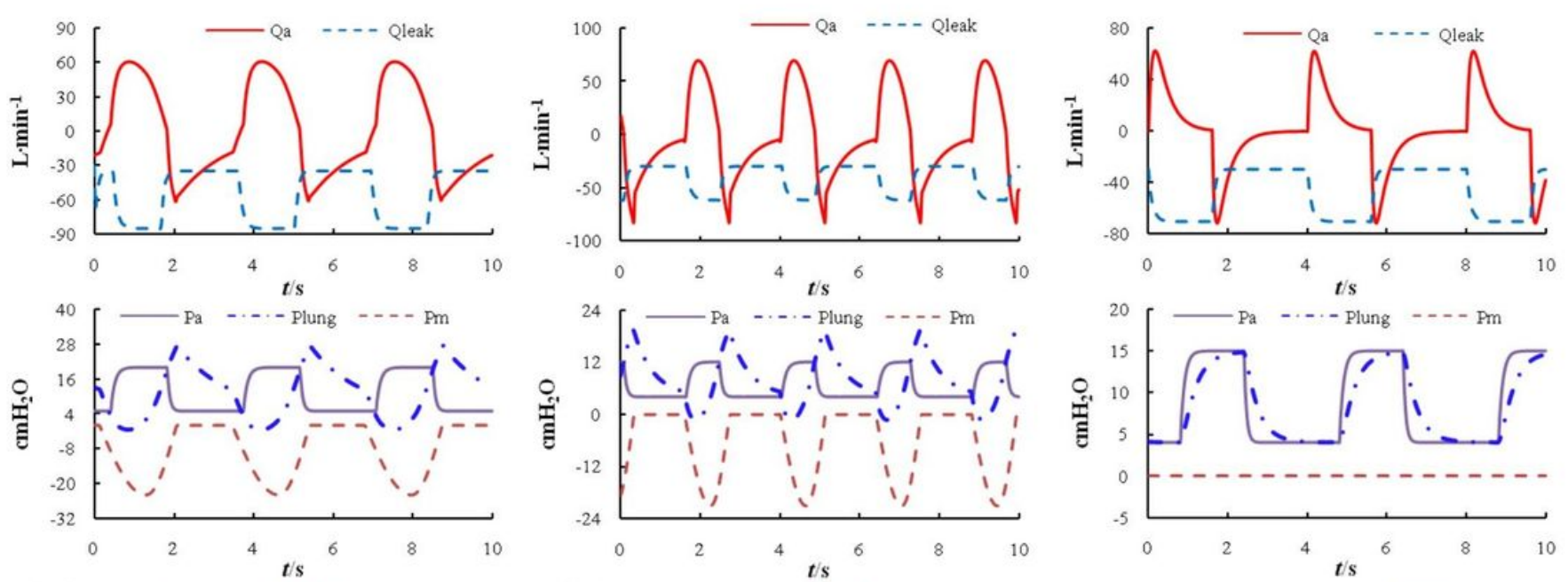

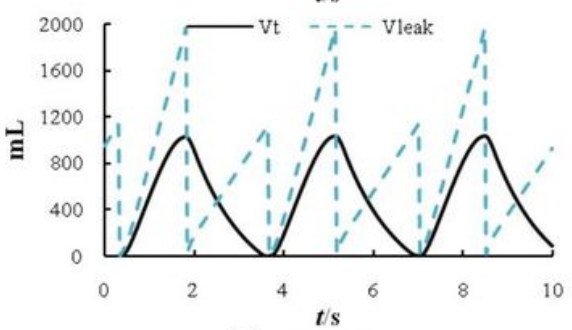

(a) COPD

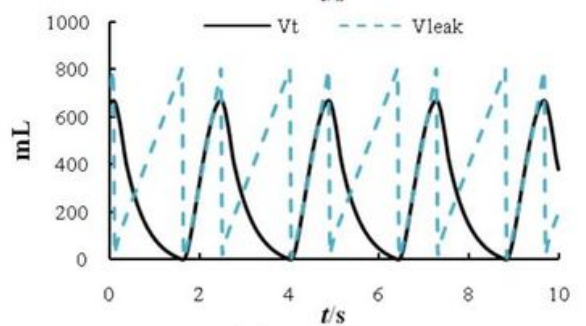

(b) ARDS

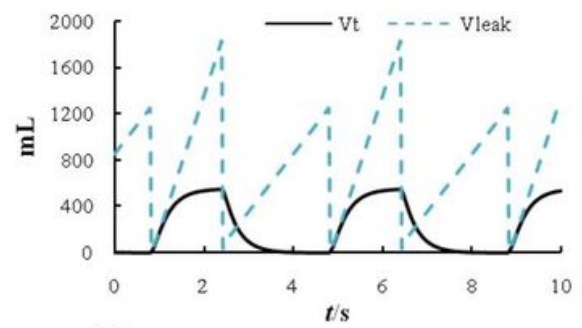

(c) without spontaneous breathing

\section{Figure 1}

the data curves of output in simulated experiments: respiratory flow (Qa), leak flow (Qleak), pressure in airway $(\mathrm{Pa})$, intrapulmonary pressure (Plung), inspiratory effort pressure (Pm), tidal volume ( $\mathrm{Vt}$ ) and leak volume (Vleak) 

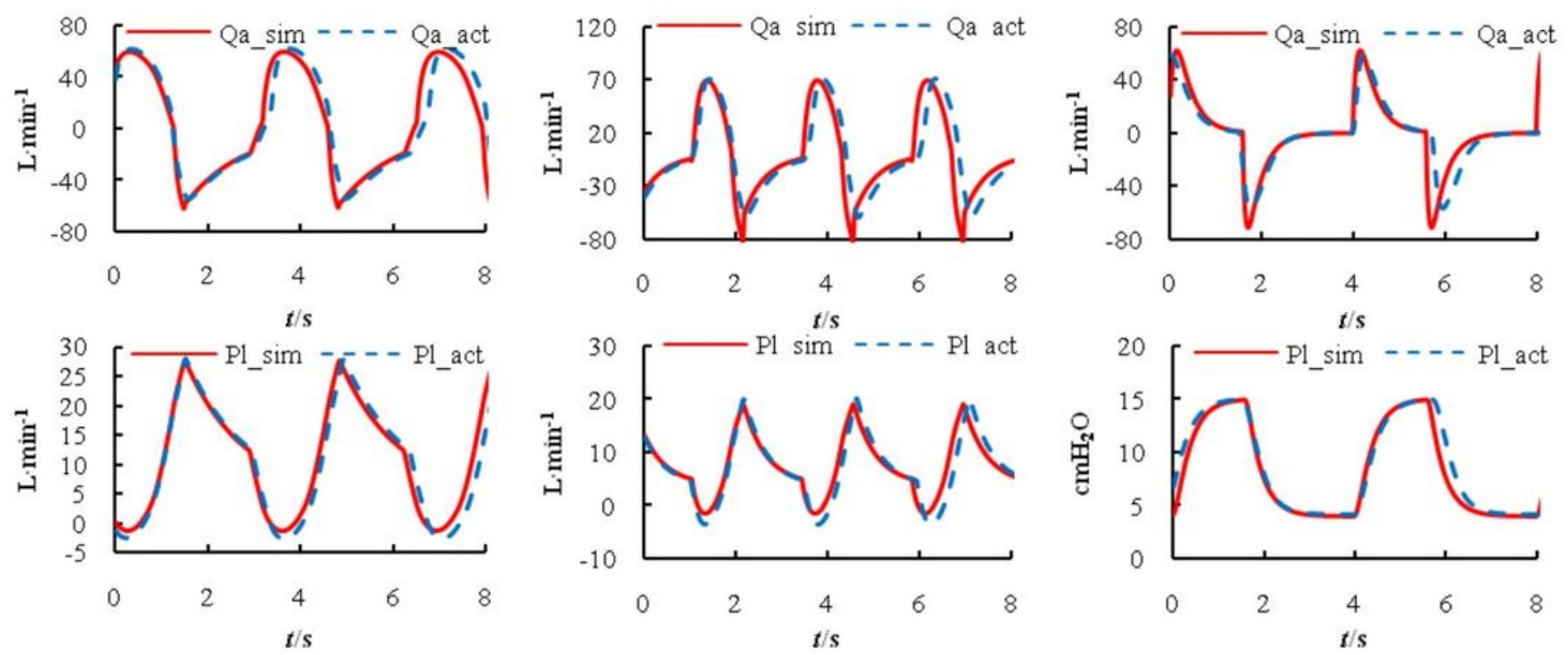

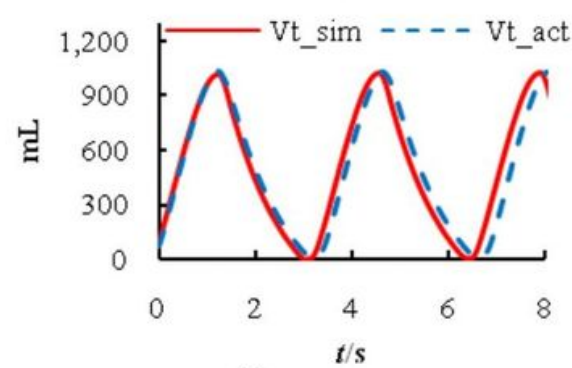

(a) COPD

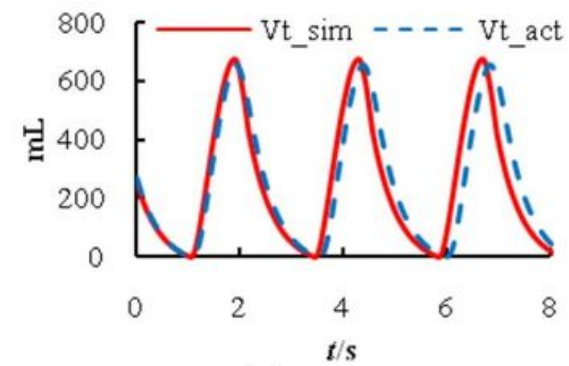

(b) ARDS

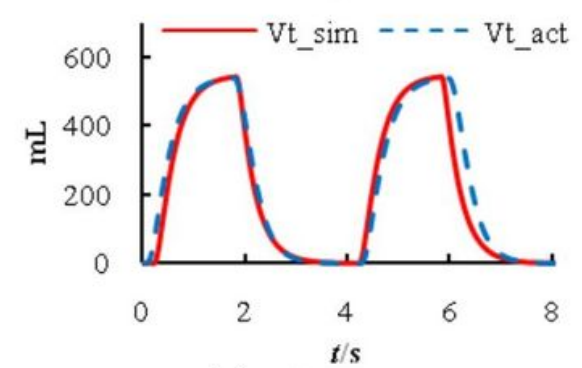

(c) without SB

\section{Figure 2}

the data collected in simulations compared to the data collected in physical experiments: simulated respiratory flow in simulation (Qa_sim), physical experimental respiratory flow (Qa_act), simulated intrapulmonary pressure (PI_sim), physical experimental intrapulmonary pressure (PI_act), simulated tidal volume (Vt_sim) and physical experimental tidal volume (Vt_act) 


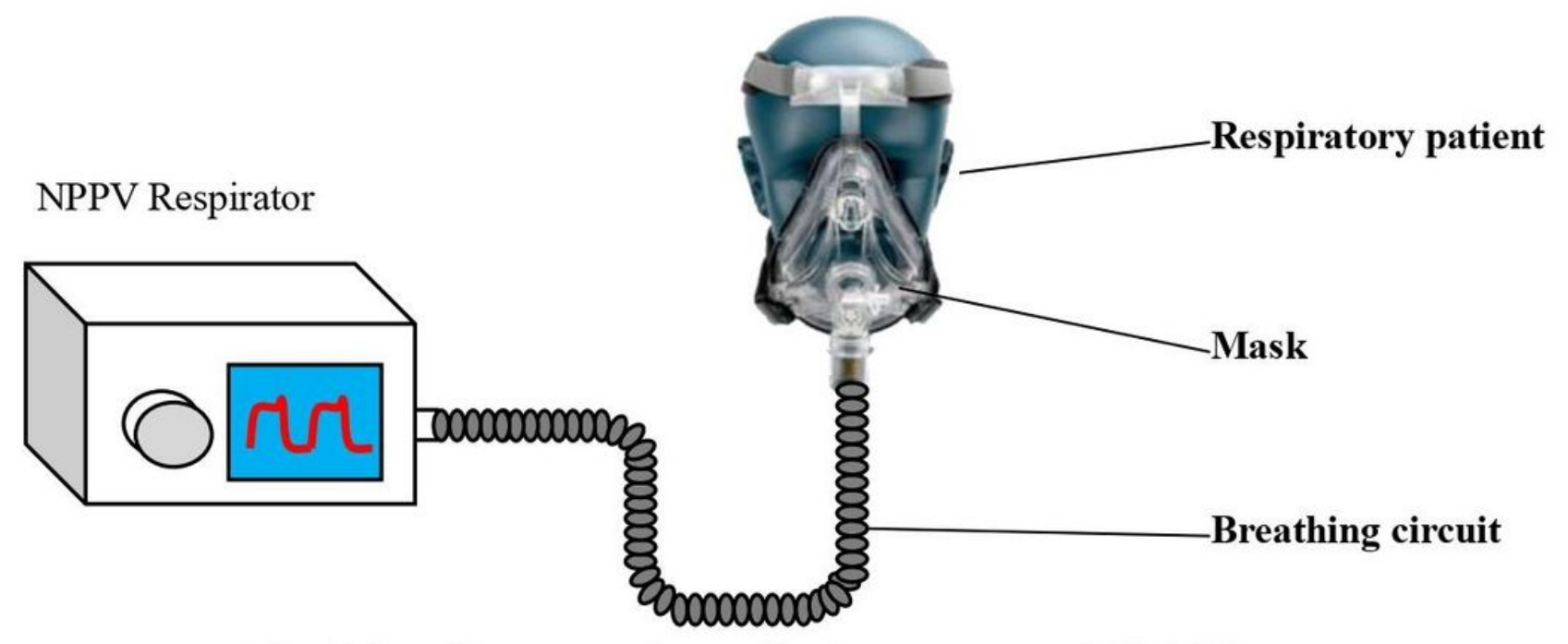

Figure 3

the diagram of ventilation system of NPPV

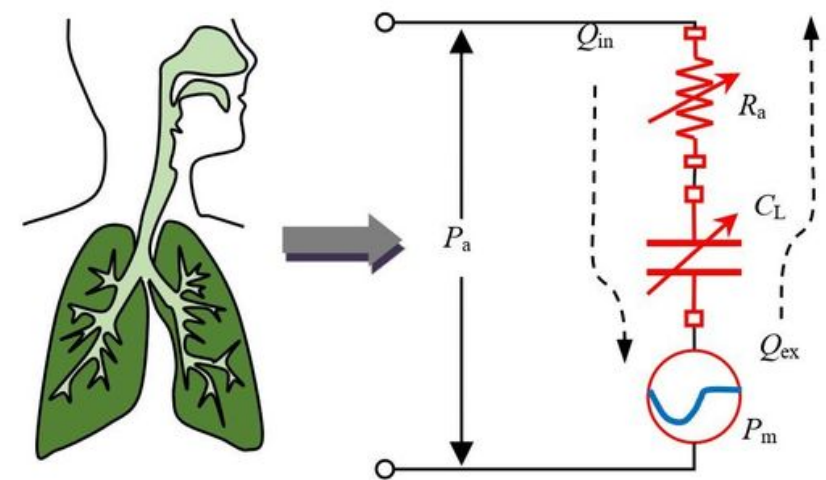

(a) human respiratorv model

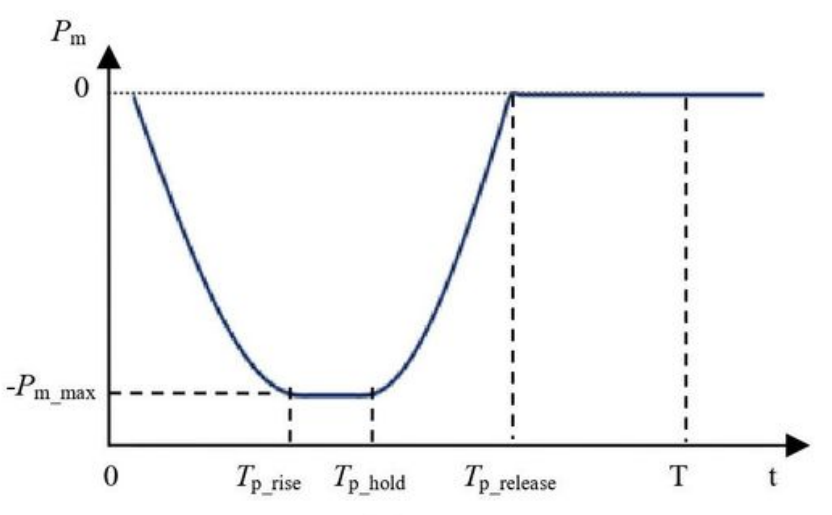

（b） curve of $P_{\mathrm{m}}$

Figure 4

the model of respiratory and effort curve 


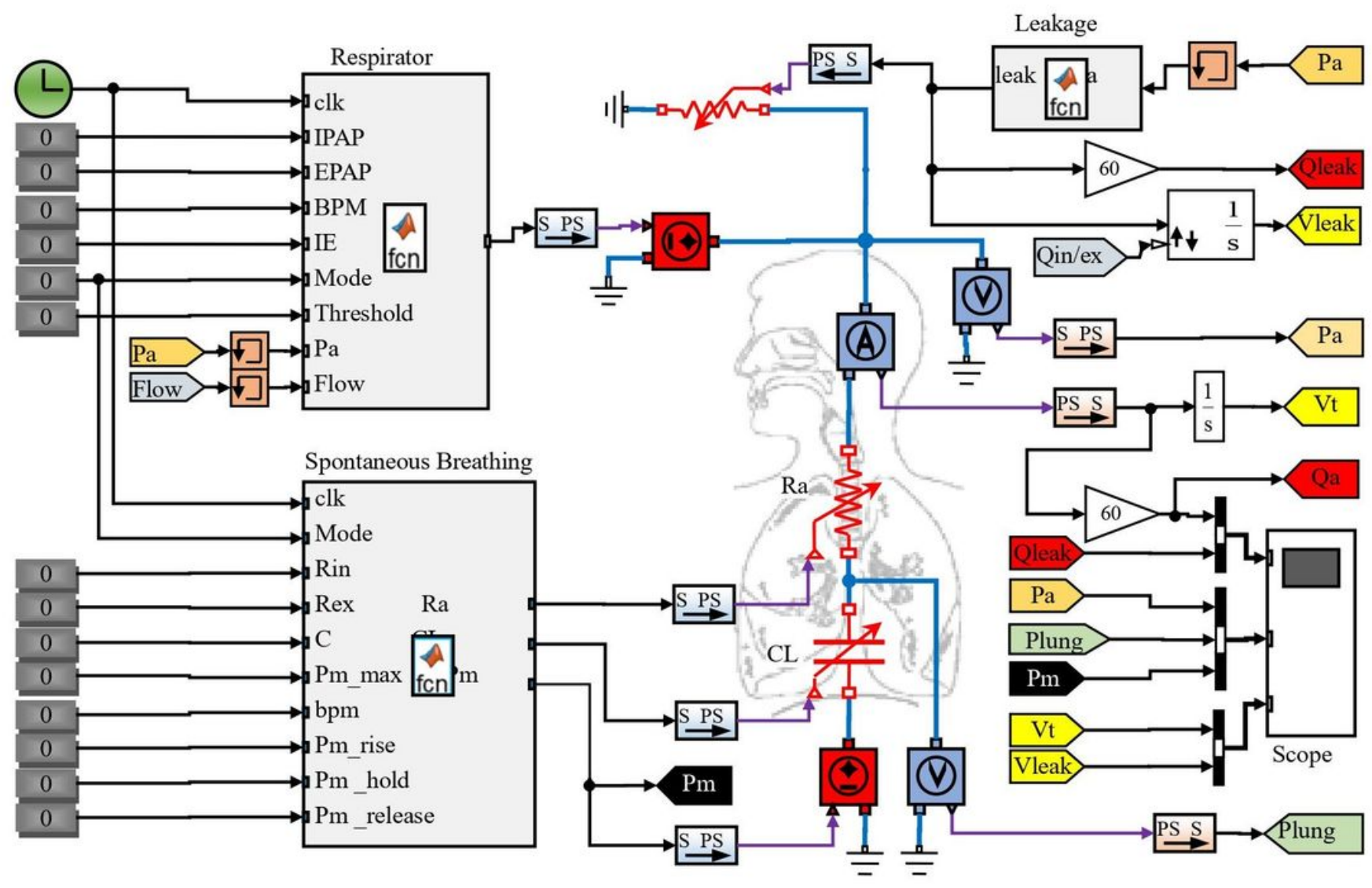

Figure 5

the simulation platform of ventilation system 


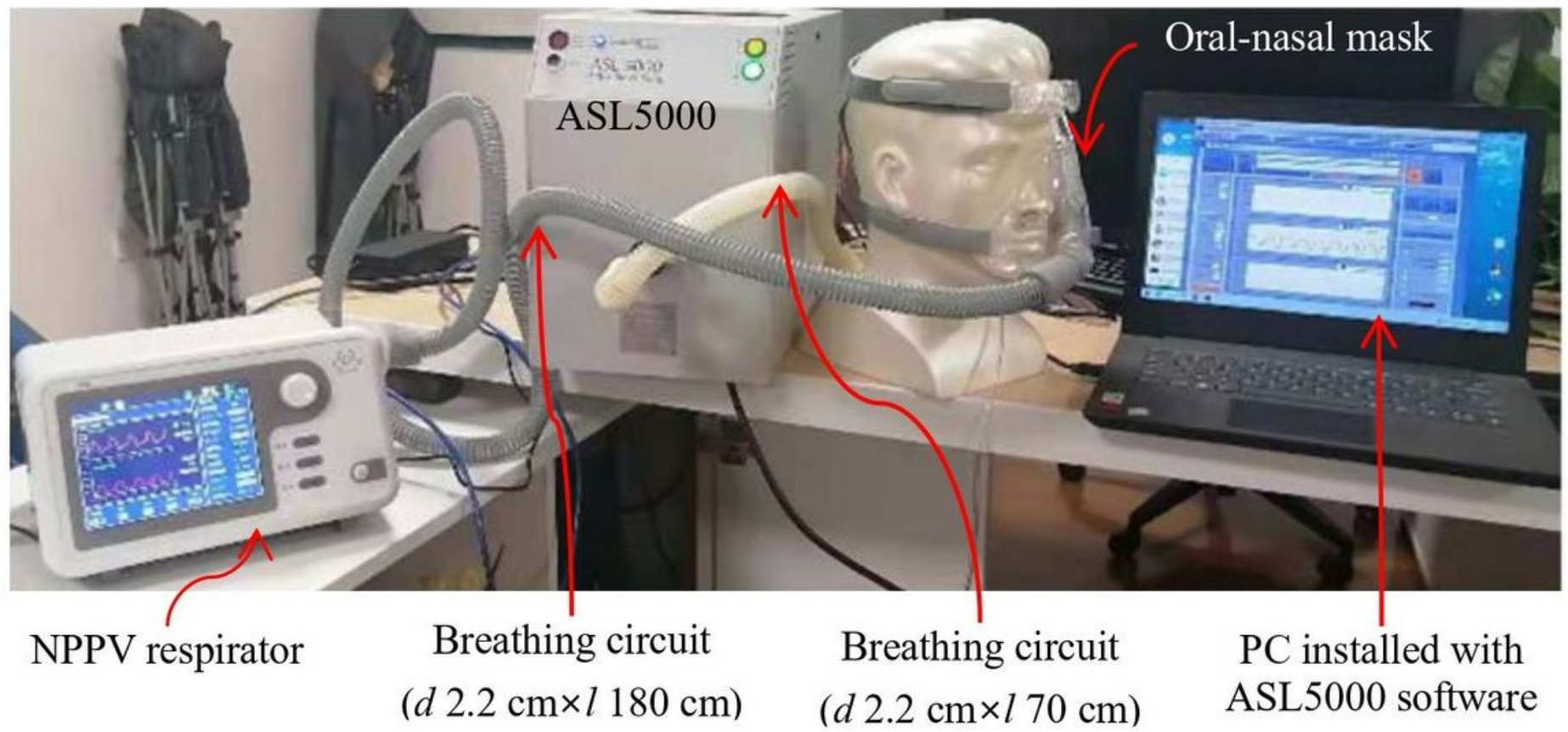

Figure 6

the physical test platform 